\title{
On Fuzzy Generalized b-connected Space in Fuzzy Topological Spaces on FuzzySet
}

\author{
Munir Abdul-Khalik Al-Khafaji ${ }^{1}$ and Ja'afer JabbarQasim ${ }^{2}$ \\ ${ }^{(1)}$ Mathematics Department, College of Education / University of Al-Mustansiriya, Iraq. \\ ${ }^{(2)}$ Mathematics Department, College of Education / University of Al-Mustansiriya, Iraq.
}

\begin{abstract}
In this paper we introduce and study fuzzy generalized b-connected spacein fuzzy Topological space on fuzzy sets and introduce some types of fuzzy (gp-connected,gs-connected,g $\alpha$-connected and gsp-connected) space with some properties, relations andTheorems about this subject.
\end{abstract}

Keywords:fgb-separated sets, fgb-connected space.

\section{Introduction}

The concept of fuzzy sets and fuzzy sets operation were first introduced by Zadeh [7] in 1965 The concepts of fuzzy topological space is study by Chang [1] in 1968.AndFuzzy generalized semi-Connected space is study by Fath Alla [3] in 2004 and fuzzyGeneralized semi pre-Connected space is study by Santhi, R.and D.Jayanthi[4] in 2012,in this paper we Introduce and study fuzzy generalized b-Connected space with some relations between them fuzzy (gp-connected,gs- connected,ga- connected and gsp - connected ) space.

\section{Basic Definitions}

Definition (2.1): Let $(\tilde{A}, \tilde{T})$ be a fuzzy topological space (f.t.s)and $\tilde{B}, \tilde{C}$ are fuzzy sets in $\tilde{A}$, then $\tilde{B}$, and $\tilde{C}$ are said to be :

(1) Fuzzy gb - separated iff $\operatorname{Min}\left\{\mu_{\mathrm{gbcl}(\tilde{\mathrm{B}})}(\mathrm{x}), \mu_{\tilde{\mathrm{C}}}(\mathrm{x})\right\}=0$ and $\operatorname{Min}\left\{\mu_{\mathrm{gbcl}(\tilde{\mathrm{C}})}(\mathrm{x}), \mu_{\tilde{\mathrm{B}}}(\mathrm{x})\right\}=0$

(2) Fuzzy $\mathrm{g} \alpha$ - separated iff $\operatorname{Min}\left\{\mu_{\operatorname{gacl}(\tilde{\mathrm{B}})}(\mathrm{x}), \mu_{\tilde{\mathrm{C}}}(\mathrm{x})\right\}=0$ and $\operatorname{Min}\left\{\mu_{\mathrm{gacl}(\tilde{\mathrm{C}})}(\mathrm{x}), \mu_{\tilde{\mathrm{B}}}(\mathrm{x})\right\}=0,[6]$

(3) Fuzzy gp - separated iff $\operatorname{Min}\left\{\mu_{\text {gpcl( } \tilde{\mathrm{B}})}(\mathrm{x}), \mu_{\tilde{\mathrm{C}}}(\mathrm{x})\right\}=0$ and $\operatorname{Min}\left\{\mu_{\mathrm{gpcl}(\tilde{\mathrm{C}})}(\mathrm{x}), \mu_{\tilde{\mathrm{B}}}(\mathrm{x})\right\}=0$, [8]

(4) Fuzzy gs - separated iff $\operatorname{Min}\left\{\mu_{\mathrm{gscl}(\tilde{\mathrm{B}})}(\mathrm{x}), \mu_{\tilde{\mathrm{C}}}(\mathrm{x})\right\}=0$ and $\operatorname{Min}\left\{\mu_{\mathrm{gscl}(\tilde{\mathrm{C}})}(\mathrm{x}), \mu_{\tilde{\mathrm{B}}}(\mathrm{x})\right\}=0$, [3]

(5) Fuzzy gsp - separated iff $\operatorname{Min}\left\{\mu_{\mathrm{gspcl}(\tilde{\mathrm{B}})}(\mathrm{x}), \mu_{\tilde{\mathrm{C}}}(\mathrm{x})\right\}=0$ and $\operatorname{Min}\left\{\mu_{\mathrm{gspcl}(\tilde{\mathrm{C}})}(\mathrm{x}), \mu_{\tilde{\mathrm{B}}}(\mathrm{x})\right\}=0$

\section{Definition (2.2)}

1. A fuzzy topological space $(\tilde{\mathrm{A}}, \tilde{\mathrm{T}})$ is said to be fuzzy g $\alpha$-connected if there is no proper non-empty maximal fuzzy g $\alpha$-separated sets $\widetilde{\mathrm{B}}$ and $\widetilde{\mathrm{C}}$ in $\widetilde{\mathrm{A}}$ such that $\mu_{\tilde{\mathrm{A}}}(\mathrm{x})=\operatorname{Max}\left\{\left(\mu_{\tilde{\mathrm{C}}}(\mathrm{x}),\left(\mu_{\tilde{\mathrm{B}}}(\mathrm{x})\right\}\right.\right.$. If $(\tilde{\mathrm{A}}, \tilde{\mathrm{T}})$ is not fuzzy $\mathrm{g} \alpha$-connected then it is said to be fuzzy $g \alpha-$ disconnected space.[6]

2. A fuzzy topological space $(\tilde{\mathrm{A}}, \tilde{\mathrm{T}})$ is said to be fuzzy gs-connected if there is no proper non-empty maximal fuzzy gs-separated sets $\widetilde{B}$ and $\widetilde{C}$ in $\widetilde{A}$ such that $\mu_{\tilde{\mathrm{A}}}(\mathrm{x})=\operatorname{Max}\left\{\left(\mu_{\tilde{C}}(\mathrm{x}),\left(\mu_{\tilde{\mathrm{B}}}(\mathrm{x})\right\}\right.\right.$. If $(\tilde{\mathrm{A}}, \tilde{\mathrm{T}})$ is not fuzzy gs-connected then it is said to be fuzzy

gs-disconnected space.[3]

3. A fuzzy topological space $(\tilde{\mathrm{A}}, \tilde{\mathrm{T}})$ is said to be fuzzy gp-connected if there is no proper non-empty maximal fuzzy gp-separated sets $\widetilde{B}$ and $\widetilde{C}$ in $\tilde{A}$ such that $\mu_{\tilde{A}}(x)=\operatorname{Max}\left\{\left(\mu_{\tilde{C}}(x),\left(\mu_{\tilde{B}}(x)\right\}\right.\right.$. If $(\tilde{A}, \tilde{T})$ is not fuzzy gp-connected then it is said to be fuzzy gp- disconnected space.

4. A fuzzy topological space $(\tilde{\mathrm{A}}, \tilde{\mathrm{T}})$ is said to be fuzzy gsp-connected if there is no proper non-empty maximal fuzzy gsp-separated sets $\widetilde{B}$ and $\widetilde{C}$ in $\widetilde{A}$ such that $\mu_{\tilde{\mathrm{A}}}(\mathrm{x})=\operatorname{Max}\left\{\left(\mu_{\tilde{\mathrm{C}}}(\mathrm{x}),\left(\mu_{\tilde{\mathrm{B}}}(\mathrm{x})\right\}\right.\right.$. If $(\tilde{\mathrm{A}}, \tilde{\mathrm{T}})$ is not fuzzy gsp-connected then it is said to be fuzzy gsp- disconnected space.[4]

Definition (2.3)[2]:A fuzzy set $\widetilde{B}$ in $(\tilde{A}, \tilde{T})$ is said to be fuzzy gb-clopen if and only if both fuzzy gb-open and fuzzy gb-closed set .

Definition (2.4)[3]:Let $\tilde{B}$ be a fuzzy set in $\tilde{A}$, then $\tilde{B}$ is said to be maximal fuzzy set in $\tilde{A}$ if for each $\mathrm{x} \in \mathrm{X} \mu_{\tilde{\mathrm{B}}}(\mathrm{x}) \neq 0$, then $\mu_{\tilde{\mathrm{B}}}(\mathrm{x})=\mu_{\tilde{\mathrm{A}}}(\mathrm{x})$

Proposition (2.5) [4]:Let $\tilde{\mathrm{B}}, \tilde{\mathrm{C}}$ be any fuzzy sets in $(\tilde{\mathrm{A}}, \tilde{\mathrm{T}})$, then

1. $\quad \tilde{\mathrm{B}} \tilde{\mathrm{q}} \tilde{\mathrm{C}} \Longrightarrow \mu_{\tilde{\mathrm{B}}}(\mathrm{x}) \leq \mu_{\tilde{\mathrm{C}}^{\mathrm{c}}}(\mathrm{x})$ or $\mu_{\tilde{\mathrm{C}}}(\mathrm{x}) \leq \mu_{\tilde{\mathrm{B}}^{\mathrm{c}}}(\mathrm{x})$.

2. $\quad \mathrm{x}_{\mathrm{r}} \tilde{\mathrm{q}} \tilde{\mathrm{B}} \Leftrightarrow \mu_{\mathrm{x}_{\mathrm{r}}}(\mathrm{x}) \leq \mu_{\tilde{\mathrm{B}}^{c}}(\mathrm{x})$. 
3. $\quad \tilde{\mathrm{B}} \tilde{\mathrm{q}} \tilde{\mathrm{B}}^{\mathrm{c}}$, for any fuzzy set $\tilde{\mathrm{B}}$ in $\tilde{\mathrm{A}}$.

4. If Min $\left\{\mu_{\tilde{\mathrm{B}}(\mathrm{x})}, \mu_{\tilde{\mathrm{C}}(\mathrm{x})}\right\}=\mu_{\tilde{\Phi}(\mathrm{x})}$, then $\mu_{\tilde{\mathrm{B}}(\mathrm{x})}+\mu_{\tilde{\mathrm{C}}(\mathrm{x})} \leq \mu_{\tilde{\mathrm{A}}(\mathrm{x})}$.

5. $\quad \operatorname{Min}\left\{\left(\mu_{\tilde{\mathrm{C}}}(\mathrm{x}),\left(\mu_{\tilde{\mathrm{B}}}(\mathrm{x})\right\}=\mu_{\tilde{\emptyset}}(\mathrm{x}) \Rightarrow \mu_{\tilde{\mathrm{C}}}(\mathrm{x}) \leq \mu_{\tilde{\mathrm{B}}}^{\mathrm{c}}(\mathrm{x})\right.\right.$ or $\mu_{\tilde{\mathrm{B}}}(\mathrm{x}) \leq \mu_{\tilde{\mathrm{C}}}^{\mathrm{c}}(\mathrm{x})$.

Theorem (2.6)[6]: If $(\tilde{\mathrm{A}}, \tilde{\mathrm{T}})$ is a f.t.s, $\tilde{\mathrm{B}}$ and $\widetilde{\mathrm{C}}$ are fuzzy sets in $\tilde{\mathrm{A}}$, then:

1. If $\tilde{\mathrm{B}} \cap \widetilde{\mathrm{C}}=\tilde{\phi}, \tilde{\mathrm{B}}$ and $\widetilde{\mathrm{C}}$ are fuzzy gb-closed sets in $\tilde{\mathrm{A}}$, then $\tilde{\mathrm{B}}$ and $\widetilde{\mathrm{C}}$ are fuzzy gb-separated in $\tilde{\mathrm{A}}$.

2. If $\tilde{\mathrm{B}} \cap \widetilde{\mathrm{C}}=\tilde{\phi}, \tilde{\mathrm{B}}$ and $\widetilde{\mathrm{C}}$ are fuzzy gb-open sets in $\tilde{\mathrm{A}}$, then $\tilde{\mathrm{B}}$ and $\widetilde{\mathrm{C}}$ are fuzzy weak gb-separated in $\tilde{\mathrm{A}}$.

\section{Theorem (2.7)}

1- If $(\tilde{\mathrm{A}}, \tilde{\mathrm{T}})$ is a f.t.s and $\tilde{\mathrm{B}}, \tilde{\mathrm{C}}$ are Fg$\alpha-$ separated in $\tilde{\mathrm{A}}$ then $\tilde{\mathrm{B}}$ and $\tilde{\mathrm{C}}$ are Fgp- separated .

2- If $(\tilde{\mathrm{A}}, \tilde{\mathrm{T}})$ is a f.t.s and $\tilde{\mathrm{B}}, \tilde{\mathrm{C}}$ are Fgo- separated in $\tilde{\mathrm{A}}$ then $\tilde{\mathrm{B}}$ and $\tilde{\mathrm{C}}$ are Fgs- separated .

3- If $(\tilde{\mathrm{A}}, \tilde{\mathrm{T}})$ is a f.t.s and $\tilde{\mathrm{B}}, \tilde{\mathrm{C}}$ are Fgo- separated in $\tilde{\mathrm{A}}$ then $\tilde{\mathrm{B}}$ and $\tilde{\mathrm{C}}$ are Fgb- separated .

4- If $(\tilde{\mathrm{A}}, \tilde{\mathrm{T}})$ is a f.t.s and $\tilde{\mathrm{B}}, \tilde{\mathrm{C}}$ are Fgp- separated in $\tilde{\mathrm{A}}$ then $\tilde{\mathrm{B}}$ and $\tilde{\mathrm{C}}$ are Fgb- separated .

5- If $(\tilde{\mathrm{A}}, \tilde{\mathrm{T}})$ is a f.t.s and $\tilde{\mathrm{B}}, \tilde{\mathrm{C}}$ are Fgs- separated in $\tilde{\mathrm{A}}$ then $\tilde{\mathrm{B}}$ and $\tilde{\mathrm{C}}$ are Fgb- separated .

6- If $(\tilde{\mathrm{A}}, \tilde{\mathrm{T}})$ is a f.t.s and $\tilde{\mathrm{B}}, \tilde{\mathrm{C}}$ are Fgp- separated in $\tilde{\mathrm{A}}$ then $\tilde{\mathrm{B}}$ and $\tilde{\mathrm{C}}$ are Fgsp- separated

7- If $(\tilde{\mathrm{A}}, \tilde{\mathrm{T}})$ is a f.t.s and $\tilde{\mathrm{B}}, \tilde{\mathrm{C}}$ are Fgs- separated in $\tilde{\mathrm{A}}$ then $\tilde{B}$ and $\tilde{\mathrm{C}}$ are Fgsp- separated .

8- If $(\tilde{\mathrm{A}}, \tilde{\mathrm{T}})$ is a f.t.s and $\tilde{\mathrm{B}}, \tilde{\mathrm{C}}$ are Fgb- separated in $\tilde{\mathrm{A}}$ then $\tilde{\mathrm{B}}$ and $\tilde{\mathrm{C}}$ are Fgsp- separated .

Proof (1)

Since $\tilde{B}$ and $\tilde{C}$ are Fg $\quad \alpha-\quad$ separated in $\tilde{A} \quad, \quad$ that implies, Min $\left\{\mu_{\operatorname{gacl}(\tilde{B})}(\mathrm{x}) \quad, \mu \tilde{\mathrm{C}}(\mathrm{x})\right\}=0 \quad$ and $\operatorname{Min}\left\{\mu_{\operatorname{gacl}(\tilde{C})}(\mathrm{x}), \mu_{\tilde{\mathrm{B}}}(\mathrm{x})\right\}=0$.

Since $\operatorname{gacl}(\tilde{\mathrm{B}})$ and $\operatorname{gacl}(\tilde{\mathrm{C}})$ are $\mathrm{Fg} \alpha$ - closed sets in $\tilde{\mathrm{A}}$. Then

$\operatorname{Min}\left\{\mu_{\text {gpcl( }(\tilde{\mathrm{B}})}(\mathrm{x}), \mu_{\tilde{\mathrm{C}}}(\mathrm{x})\right\}=0$ and $\operatorname{Min}\left\{\mu_{\mathrm{gpcl}(\tilde{\mathrm{C}})}(\mathrm{x}), \mu_{\tilde{\mathrm{B}}}(\mathrm{x})\right\}=0$

Hence, $\mathrm{B}$ and $\tilde{\mathrm{C}}$ are Fgp- separated in $\tilde{\mathrm{A}}$

The $\operatorname{proof}(2,3,4,5,6,7,8)$ is similar to that of (1) theorem $(2.7)$

\section{Some Properties of Fuzzy Generalized b-ConnectedSpace}

Definition (3.1):

1.A fuzzy topological space $(\tilde{\mathrm{A}}, \tilde{\mathrm{T}})$ is said to be fuzzy gb-connected if there is no proper non-empty maximal fuzzy gb-separated sets $\tilde{\mathrm{B}}$ and $\tilde{C}$ in $\tilde{A}$ suchthat $\mu_{\tilde{\mathrm{A}}}(\mathrm{x})=\operatorname{Max}\left\{\left(\mu_{\tilde{\mathrm{C}}}(\mathrm{x}),\left(\mu_{\tilde{\mathrm{B}}}(\mathrm{x})\right\}\right.\right.$. If $(\tilde{\mathrm{A}}, \tilde{\mathrm{T}})$ is not fuzzy gb-connected then it is said to be fuzzy gb-disconnected space.

2.A fuzzy topological space $(\tilde{\mathrm{A}}, \tilde{\mathrm{T}})$ is said to be fuzzy weak gb-connected fuzzy if there is no proper non-empty maximal fuzzy weak gb-separated sets $\tilde{\mathrm{B}}$ and $\tilde{\mathrm{C}}$ in $\tilde{\mathrm{A}}$ such that $\mu_{\tilde{\mathrm{A}}}(\mathrm{x})=\operatorname{Max}\left\{\left(\mu_{\tilde{\mathrm{C}}}(\mathrm{x}),\left(\mu_{\tilde{\mathrm{B}}}(\mathrm{x})\right\}\right.\right.$. If $(\tilde{\mathrm{A}}, \tilde{\mathrm{T}})$ is not fuzzy weak gb-connected is said to be fuzzy weak gb-disconnected space.[3]

Definition (3.2) [4],[5]

A fuzzy topological space ( $\tilde{A}, \tilde{T})$ is said to be fuzzy generalized b-connected(denoted by Fgb- connected) if and only if the fuzzy sets which are both Fgb-open and Fgb-closed.

Proposition (3.3):Every fuzzy gb-connected space ( $\tilde{\mathrm{A}}, \tilde{\mathrm{T}})$ is fuzzy weak gb-connected space.

Proof:

Suppose that $\tilde{\mathrm{B}}$ and $\tilde{\mathrm{C}}$ be proper non-empty maximal fuzzy gb-separated sets in $\tilde{\mathrm{A}}$

Implies that, $\tilde{B}$ and $\tilde{C}$ are proper non-empty maximal fuzzy weak gb-separated sets in $\tilde{A}$

Since $(\tilde{\mathrm{A}}, \tilde{\mathrm{T}})$ is fuzzy gb-connected space

Then, $\mu_{\tilde{\mathrm{A}}}(\mathrm{x}) \neq \operatorname{Max}\left\{\left(\mu_{\tilde{\mathrm{C}}}(\mathrm{x}),\left(\mu_{\tilde{\mathrm{B}}}(\mathrm{x})\right\}\right.\right.$.

Therefore, $(\tilde{\mathrm{A}}, \tilde{T})$ is a fuzzy weak gb-connected space.

Remark 3.4: The converse of proposition (3.3) is not true in general.

Example 3.5 :Let $X=\{a, b\}$ and $\tilde{A}, \tilde{B}, \tilde{C}, \widetilde{D}, \widetilde{E}, \widetilde{F}$ are fuzzy sets defined as follows:

$\tilde{\mathrm{A}}=\{(\mathrm{a}, 0.3),(\mathrm{b}, 0.5)\}, \tilde{\mathrm{B}}=\{(\mathrm{a}, 0),(\mathrm{b}, 0.3)\}, \tilde{\mathrm{C}}=\{(\mathrm{a}, 0.3),(\mathrm{b}, 0)\}, \widetilde{\mathrm{D}}=\{(\mathrm{a}, 0.3),(\mathrm{b}, 0.3)\}, \widetilde{\mathrm{E}}=\{(\mathrm{a}, 0.2),(\mathrm{b}, 0.2)\}, \widetilde{\mathrm{F}}=\{(\mathrm{a}, 0)$, $(\mathrm{b}, 0.1)\}$.

Let $\widetilde{T}=\{\widetilde{\phi}, \widetilde{A}, \widetilde{B}, \widetilde{C}, \widetilde{D}\}$ be a fuzzy topology on $\tilde{A}$, then $\widetilde{E}$ and $\widetilde{\mathrm{F}}$ are fuzzy weak gb-connected space but not fuzzy gb-connected space.

Theorem (3.6)

1- If $(\tilde{\mathrm{A}}, \tilde{\mathrm{T}})$ a f.t.s, $\tilde{\mathrm{B}}$ and $\tilde{\mathrm{C}}$ are fuzzy gb-connected in $\tilde{\mathrm{A}}$, then $\tilde{\mathrm{B}}$ and $\tilde{\mathrm{C}}$ are fuzzy g $\alpha$-connected space.

2- If $(\tilde{\mathrm{A}}, \tilde{\mathrm{T}})$ a f.t.s, $\tilde{B}$ and $\tilde{C}$ are fuzzy gb-connected in $\tilde{\mathrm{A}}$, then $\tilde{B}$ and $\tilde{C}$ are fuzzy gs-connected space. 
3- If $(\tilde{A}, \tilde{T})$ a f.t.s, B̈andC̃ are fuzzy gb-connected in $\tilde{A}$, then B̃and $\tilde{C}$ are fuzzy gp-connected space. 4- If $(\tilde{A}, \tilde{T})$ a f.t.s, $\tilde{B}$ and $\tilde{C}$ are fuzzy gs-connected in $\tilde{A}$, then $\tilde{B}$ and $\tilde{C}$ are fuzzy g $\alpha$-connected space. 5- If $(\tilde{\mathrm{A}}, \tilde{\mathrm{T}})$ a f.t.s, $\tilde{\mathrm{B} a n d} \tilde{\mathrm{C}}$ are fuzzy gp-connected in $\tilde{\mathrm{A}}$, then $\tilde{\mathrm{B}}$ and $\tilde{\mathrm{C}}$ are fuzzy g $\alpha$-connected space. 6- If $(\tilde{\mathrm{A}}, \tilde{\mathrm{T}})$ a f.t.s, $\tilde{\mathrm{B}}$ and $\tilde{C}$ are fuzzy gsp-connected in $\tilde{\mathrm{A}}$, then $\tilde{\mathrm{B}}$ and $\tilde{\mathrm{C}}$ are fuzzy gb-connected space. 7- If $(\tilde{\mathrm{A}}, \tilde{\mathrm{T}})$ a f.t.s, $\tilde{B}$ and $\tilde{C}$ are fuzzy gsp-connected in $\tilde{\mathrm{A}}$, then $\tilde{B}$ and $\tilde{C}$ are fuzzy gs-connected space. 8- If $(\tilde{A}, \tilde{T})$ a f.t.s, $\tilde{B}$ and $\tilde{C}$ are fuzzy gsp-connected in $\tilde{A}$, then $\tilde{B}$ and $\tilde{C}$ are fuzzy gp-connected space.

Proof (1) :Let $(\tilde{\mathrm{A}}, \tilde{\mathrm{T}})$ is fuzzy gb-connected space

Suppose that $(\tilde{\mathrm{A}}, \tilde{\mathrm{T}})$ is fuzzy $\mathrm{g} \alpha$-disconnected space

Then this implies that there exist non-empty maximal fuzzy go-separated sets $\tilde{B}$ and $\tilde{C}$ in $\tilde{A}$ such that $\tilde{\mathrm{A}}=\tilde{\mathrm{B}} \cup \tilde{\mathrm{C}}$. Then by theorem (2.7) there exist non-empty maximal fuzzy gb-separated sets $\tilde{B}$ and $\tilde{C}$ in $\tilde{\mathrm{A}}$ such that $\tilde{\mathrm{A}}=\tilde{\mathrm{B}} \cup \tilde{\mathrm{C}}$. Implies that $(\tilde{\mathrm{A}}, \tilde{\mathrm{T}})$ is fuzzy gb-disconnected which is a contradiction

Hence $(\tilde{\mathrm{A}}, \tilde{\mathrm{T}})$ is fuzzy g $\alpha$-connected space.

The Proof $(2,3,4,5,6,7,8,9)$ is similar to that of (1) theorem (3.6).

Remark(3.7): The converse of (1) of theorem (3.6) is not true in general as shown by the following example. Example (3.8):Let $\mathrm{X}=\{\mathrm{a}, \mathrm{b}\}$ and $\tilde{\mathrm{A}}, \tilde{\mathrm{B}}, \tilde{\mathrm{C}}, \tilde{\mathrm{D}}, \tilde{\mathrm{E}}$ are fuzzy sets defined as follows:

$\tilde{\mathrm{A}}=\{(\mathrm{a}, 0.8),(\mathrm{b}, 0.8)\}, \quad \tilde{\mathrm{B}}=\{(\mathrm{a}, 0.6),(\mathrm{b}, 0.6)\}, \quad \tilde{\mathrm{C}}=\{(\mathrm{a}, 0.6),(\mathrm{b}, 0.8)\}, \quad \tilde{\mathrm{D}}=\{(\mathrm{a}, 0.8),(\mathrm{b}, 0.6)\} \tilde{\mathrm{E}}=\{(\mathrm{a}, 0.0),(\mathrm{b}, 0.8)\}$, $\tilde{\mathrm{F}}=\{(\mathrm{a}, 0.8),(\mathrm{b}, 0.0)\}$, let $\tilde{\tau}=\{\tilde{\phi}, \tilde{\mathrm{A}}, \tilde{\mathrm{B}}, \tilde{\mathrm{C}}, \tilde{\mathrm{D}}\}$ be fuzzy topology on $\tilde{\mathrm{A}}$ and the $\mathrm{FG \alpha O}(\tilde{\mathrm{A}})=\{\tilde{\phi}, \tilde{\mathrm{A}}, \tilde{\mathrm{B}}, \tilde{\mathrm{C}}, \tilde{\mathrm{D}}, \tilde{\mathrm{E}}, \tilde{\mathrm{F}}\}$, then $(\tilde{\mathrm{A}}, \tilde{\tau})$ is a fuzzy g $\alpha$-connected space but not fuzzy gb-connected space.

Remark (3.9): The converse of (2) of theorem (3.6) is not true in general as shown by the following example. Example (3.10): Let $X=\{a, b\}$, and $\tilde{A}, \tilde{B}, \tilde{C}, \tilde{D}, \tilde{E}, \tilde{F}$ are fuzzy sets defined as follows:

$\tilde{\mathrm{A}}=\{(\mathrm{a}, 0.7),(\mathrm{b}, 0.7)\}, \quad \tilde{\mathrm{B}}=\{(\mathrm{a}, 0.7),(\mathrm{b}, 0.3)\}, \quad \tilde{\mathrm{C}}=\{(\mathrm{a}, 0.3),(\mathrm{b}, 0.7)\}, \tilde{\mathrm{D}}=\{(\mathrm{a}, 0.3),(\mathrm{b}, 0.3)\}, \quad \tilde{\mathrm{E}}=\{(\mathrm{a}, 0.7),(\mathrm{b}, 0.0)\}$, $\tilde{\mathrm{F}}=\{(\mathrm{a}, 0.0),(\mathrm{b}, 0.7)\}$ be a fuzzy sets in $\tilde{\mathrm{A}}$,

$\tilde{\tau}=\{\tilde{\phi}, \tilde{A}, \tilde{B}, \tilde{C}, \tilde{D}\}$ be afuzzy topology on $\tilde{A}$ and the $\operatorname{FGSO}(\tilde{\mathrm{A}})=\{\tilde{\phi}, \tilde{\mathrm{A}}, \tilde{\mathrm{B}}, \tilde{\mathrm{C}}, \tilde{D}, \tilde{E}, \tilde{\mathrm{F}}\}$ then $(\tilde{\mathrm{A}}, \tilde{\tau})$ is a fuzzy gsconnected space but not fuzzy gb-connected space.

Remark (3.11): The converse of (3) of theorem (3.6) is not true in general as shown by the following example. Example (3.12): Let $\mathrm{X}=\{\mathrm{a}, \mathrm{b}\}$ and $\tilde{\mathrm{A}}, \tilde{\mathrm{B}}_{1}, \tilde{\mathrm{B}}_{2}, \ldots, \tilde{\mathrm{B}}_{17}$ are fuzzy sets defined as follows:

$\tilde{\mathrm{A}}=\{(\mathrm{a}, 0.7),(\mathrm{b}, 0.9)\}, \widetilde{\mathrm{B}}_{1}=\{(\mathrm{a}, 0.1),(\mathrm{b}, 0.9)\}, \widetilde{\mathrm{B}}_{2}=\{(\mathrm{a}, 0.7),(\mathrm{b}, 0.1)\}, \tilde{\mathrm{B}}_{3}=\{(\mathrm{a}, 0.1),(\mathrm{b}, 0.1)\}$,

$\tilde{\mathrm{B}}_{5}=\{(\mathrm{a}, 0.5),(\mathrm{b}, 0.0)\}, \tilde{\mathrm{B}}_{6}=\{(\mathrm{a}, 0.5),(\mathrm{b}, 0.9)\}, \tilde{\mathrm{B}}_{7}=\{(\mathrm{a}, 0.1),(\mathrm{b}, 0.7)\}, \tilde{\mathrm{B}}_{8}=\{(\mathrm{a}, 0.0),(\mathrm{b}, 0.1)\}$,

$\tilde{\mathrm{B}}_{9}=\{(\mathrm{a}, 0.1),(\mathrm{b}, 0.0)\}, \tilde{\mathrm{B}}_{10}=\{(\mathrm{a}, 0.7),(\mathrm{b}, 0.8)\}, \tilde{\mathrm{B}}_{11}=\{(\mathrm{a}, 0.1),(\mathrm{b}, 0.8)\}, \tilde{\mathrm{B}}_{12}=\{(\mathrm{a}, 0.0),(\mathrm{b}, 0.7)\}$,

$\tilde{\mathrm{B}}_{13}=\{(\mathrm{a}, 0.5),(\mathrm{b}, 0.1)\}, \tilde{\mathrm{B}}_{14}=\{(\mathrm{a}, 0.7),(\mathrm{b}, 0.7)\}, \widetilde{\mathrm{B}}_{15}=\{(\mathrm{a}, 0.5),(\mathrm{b}, 0.8)\}, \widetilde{\mathrm{B}}_{16}=\{(\mathrm{a}, 0.0),(\mathrm{b}, 0.9)\}$,

$\tilde{\mathrm{B}}_{17}=\{(\mathrm{a}, 0.7),(\mathrm{b}, 0.0)\}, \mathrm{Be}$ a fuzzy sets in $\tilde{\mathrm{A}}, \tilde{\tau}=\left\{\tilde{\phi}, \tilde{\mathrm{A}}, \tilde{\mathrm{B}}_{1}, \tilde{\mathrm{B}}_{2}, \tilde{\mathrm{B}}_{3}, \tilde{\mathrm{B}}_{4}, \tilde{\mathrm{B}}_{5}, \tilde{\mathrm{B}}_{6}, \tilde{\mathrm{B}}_{7}, \tilde{\mathrm{B}}_{8}, \tilde{\mathrm{B}}_{9}, \tilde{\mathrm{B}}_{10}, \tilde{\mathrm{B}}_{11}, \tilde{\mathrm{B}}_{12}, \tilde{\mathrm{B}}_{13}, \tilde{\mathrm{B}}_{14}, \tilde{\mathrm{B}}_{15}\right\}$ be a fuzzy topology on $\tilde{\mathrm{A}}$, the $\operatorname{FGPO}(\tilde{\mathrm{A}})=\left\{\tilde{\phi}, \tilde{\mathrm{A}}, \tilde{\mathrm{B}}_{1}, \tilde{\mathrm{B}}_{2}, \tilde{\mathrm{B}}_{3}, \tilde{\mathrm{B}}_{4}, \tilde{\mathrm{B}}_{5}, \tilde{\mathrm{B}}_{6}, \tilde{\mathrm{B}}_{7}, \tilde{\mathrm{B}}_{8}, \tilde{\mathrm{B}}_{9}, \tilde{\mathrm{B}}_{10}, \tilde{\mathrm{B}}_{11}, \tilde{\mathrm{B}}_{12}, \tilde{\mathrm{B}}_{13}, \tilde{\mathrm{B}}_{15}, \tilde{\mathrm{B}}_{16}, \tilde{\mathrm{B}}_{17}\right\}$, then $(\tilde{\mathrm{A}}$, $\tilde{\tau})$ is a fuzzy gp - connected space but not fuzzy gb-connected space.

Remark (3.13): The converse of (4) of theorem (3.6) is not true in general as shown by the following example. Example (3.14): Let $X=\{a, b\}$ and $\tilde{A}, \tilde{B}, \tilde{C}, \tilde{D}, \tilde{E}, \tilde{F}$ are fuzzy sets defined as follows:

$\tilde{\mathrm{A}}=\{(\mathrm{a}, 0.5),(\mathrm{b}, 0.5)\}, \quad \tilde{\mathrm{B}}=\{(\mathrm{a}, 0.3),(\mathrm{b}, 0.5)\}, \quad \tilde{\mathrm{C}}=\{(\mathrm{a}, 0.5),(\mathrm{b}, 0.3)\}, \quad \tilde{\mathrm{D}}=\{(\mathrm{a}, 0.3),(\mathrm{b}, 0.3)\}, \quad \tilde{\mathrm{E}}=\{(\mathrm{a}, 0.5),(\mathrm{b}, 0.0)\}$, $\tilde{\mathrm{F}}=\{(\mathrm{a}, 0.0),(\mathrm{b}, 0.5)\}$, let $\tilde{\mathrm{T}}=\{\tilde{\mathrm{A}}, \tilde{\varnothing}, \tilde{\mathrm{B}}, \tilde{\mathrm{C}}, \tilde{\mathrm{D}}\}$ be fuzzy Topology on $\tilde{\mathrm{A}}$ and the $\mathrm{FG} \alpha \mathrm{O}(\tilde{\mathrm{A}})=\{\tilde{\phi}, \tilde{\mathrm{A}}, \tilde{\mathrm{B}}, \tilde{\mathrm{C}}, \tilde{\mathrm{D}}, \tilde{\mathrm{E}}, \tilde{\mathrm{F}}\}$, then $(\tilde{\mathrm{A}}$ $, \tilde{\tau})$ is a fuzzy $g \alpha$-connected space but not fuzzy gs-connected space.

Remark (3.15): The converse of (5) of theorem (3.6) is not true in general as shown by the following example. Example (3.16): Let $X=\{a, b\}$, and $\tilde{A}, \tilde{B}, \tilde{C}, \tilde{D}, \tilde{E}, \tilde{F}$, are fuzzy sets defined as follows:

$\tilde{\mathrm{A}}\{(\mathrm{a}, 0.6),(\mathrm{b}, 0.6)\}, \quad \tilde{\mathrm{B}}=\{(\mathrm{a}, 0.3),(\mathrm{b}, 0.6)\}, \quad \tilde{\mathrm{C}}=\{(\mathrm{a}, 0.6),(\mathrm{b}, 0.3)\}, \quad \tilde{\mathrm{D}}=\{(\mathrm{a}, 0.3),(\mathrm{b}, 0.3)\}, \quad \tilde{\mathrm{E}}=\{(\mathrm{a}, 0.6),(\mathrm{b}, 0.0)\}$, $\tilde{\mathrm{F}}=\{(\mathrm{a}, 0.0),(\mathrm{b}, 0.6)\}$ be a fuzzy sets in $\tilde{\mathrm{A}}, \tilde{\tau}=\{\tilde{\phi}, \tilde{\mathrm{A}}, \tilde{\mathrm{B}}, \tilde{\mathrm{C}}, \tilde{\mathrm{D}}\}$ be a fuzzy topology on $\tilde{\mathrm{A}}$ and the $\operatorname{FG\alpha O}(\tilde{\mathrm{A}})=\{\tilde{\phi}$ , $\tilde{\mathrm{A}}, \tilde{\mathrm{B}}, \tilde{\mathrm{C}}, \tilde{\mathrm{D}}, \tilde{\mathrm{E}}, \tilde{\mathrm{F}}\}$, then $(\tilde{\mathrm{A}}, \tilde{\tau})$ is a fuzzy g $\alpha$-connected space but not fuzzy gp-connected space.

Remark (3.17): The converse of (6) of theorem (3.6) is not true in general as shown by the following example. Example (3.18): Let $X=\{a, b\}$ and $\tilde{A}, \tilde{B}, \tilde{C}, \tilde{D}, \tilde{E}, \tilde{F}$ are fuzzy sets defined as follows:

$\tilde{\mathrm{A}}=\{(\mathrm{a}, 0.7),(\mathrm{b}, 0.9)\}, \tilde{\mathrm{B}}_{1}=\{(\mathrm{a}, 0.1),(\mathrm{b}, 0.9)\}, \tilde{\mathrm{B}}_{2}=\{(\mathrm{a}, 0.7),(\mathrm{b}, 0.1)\}$,

$\tilde{\mathrm{B}}_{3}=\{(\mathrm{a}, 0.1),(\mathrm{b}, 0.1)\}, \tilde{\mathrm{B}}_{4}=\{(\mathrm{a}, 0.0),(\mathrm{b}, 0.8)\}, \tilde{\mathrm{B}}_{5}=\{(\mathrm{a}, 0.7),(\mathrm{b}, 0.0)\}, \tilde{\mathrm{B}}_{6}=\{(\mathrm{a}, 0.7),(\mathrm{b}, 0.7)\}, \tilde{\mathrm{B}}_{7}=\{(\mathrm{a}, 0.1),(\mathrm{b}, 0.7)\}, \tilde{\mathrm{B}}_{8}=\{(\mathrm{a}, 0$ 
$.0),(\mathrm{b}, 0.1)\}, \tilde{\mathrm{B}}_{9}=\{(\mathrm{a}, 0.1),(\mathrm{b}, 0.0)\}, \tilde{\mathrm{B}}_{10}=\{(\mathrm{a}, 0.0),(\mathrm{b}, 0.1)\}, \tilde{\mathrm{B}}_{11}=\{(\mathrm{a}, 0.7),(\mathrm{b}, 0.8)\}, \tilde{\mathrm{B}}_{12}=\{(\mathrm{a}, 0.1),(\mathrm{b}, 0.8)\}, \tilde{\mathrm{B}}_{13}=\{(\mathrm{a}, 0.0),(\mathrm{b}$ $, 0.7)\}, \tilde{\mathrm{B}}_{14}=\{(\mathrm{a}, 0.0),(\mathrm{b}, 0.9)\}, \tilde{\mathrm{B}}_{15}=\{(\mathrm{a}, 0.7),(\mathrm{b}, 0.0)\}$,

letbe $\tilde{\tau}=\left\{\tilde{\phi}, \tilde{\mathrm{A}}, \tilde{\mathrm{B}}_{1}, \tilde{\mathrm{B}}_{2}, \tilde{\mathrm{B}}_{3}, \tilde{\mathrm{B}}_{4}, \tilde{\mathrm{B}}_{5}, \tilde{\mathrm{B}}_{6}, \tilde{\mathrm{B}}_{7}, \tilde{\mathrm{B}}_{8}, \tilde{\mathrm{B}}_{9}, \tilde{\mathrm{B}}_{10}, \tilde{\mathrm{B}}_{11}, \tilde{\mathrm{B}}_{12}, \tilde{\mathrm{B}}_{13}\right\}$ be a fuzzy topology on $\tilde{\mathrm{A}}$ and the $\operatorname{FGBO}(\tilde{\mathrm{A}})=\left\{\tilde{\phi}, \tilde{\mathrm{A}}, \tilde{\mathrm{B}}_{1}, \tilde{\mathrm{B}}_{2}, \tilde{\mathrm{B}}_{3}, \tilde{\mathrm{B}}_{4}, \tilde{\mathrm{B}}_{5}, \tilde{\mathrm{B}}_{6}, \tilde{\mathrm{B}}_{7}, \tilde{\mathrm{B}}_{8}, \tilde{\mathrm{B}}_{9}, \tilde{\mathrm{B}}_{10}, \tilde{\mathrm{B}}_{11}, \tilde{\mathrm{B}}_{12}, \tilde{\mathrm{B}}_{13}, \tilde{\mathrm{B}}_{15}\right\}$ Then $(\tilde{\mathrm{A}}, \tilde{\tau})$ is a fuzzy gb-connected space but not fuzzy gsp-connected space.

Remark (3.19): The converse of (7) of theorem (3.6) is not true in general as shown by the following example. Example (3.20): $\tilde{\mathrm{A}}=\{(\mathrm{a}, 0.7),(\mathrm{b}, 0.7)\}, \tilde{\mathrm{B}}=\{(\mathrm{a}, 0.4),(\mathrm{b}, 0.7)\}, \tilde{\mathrm{C}}=\{(\mathrm{a}, 0.7),(\mathrm{b}, 0.4)\}, \tilde{\mathrm{D}}=\{(\mathrm{a}, 0.4),(\mathrm{b}, 0.4)\}$, $\tilde{\mathrm{E}}=\{(\mathrm{a}, 0.0),(\mathrm{b}, 0.2)\}, \tilde{\mathrm{F}}=\{(\mathrm{a}, 0.2),(\mathrm{b}, 0.0)\}, \tilde{\mathrm{G}}=\{(\mathrm{a}, 0.2),(\mathrm{b}, 0.2)\}, \tilde{\mathrm{H}}=\{(\mathrm{a}, 0.0),(\mathrm{b}, 0.7)\}, \tilde{\mathrm{I}}=\{(\mathrm{a}, 0.7),(\mathrm{b}, 0.0)\}$, Let $\tilde{\tau}=\{\tilde{\phi}$ , $\tilde{\mathrm{A}}, \tilde{\mathrm{B}}, \tilde{\mathrm{C}}, \tilde{\mathrm{D}}, \tilde{\mathrm{E}}, \tilde{\mathrm{F}}, \tilde{\mathrm{G}}\}$ be fuzzy topology on $\tilde{\mathrm{A}}$ and the $\operatorname{FGSO}(\tilde{\mathrm{A}})=\{\tilde{\phi}, \tilde{\mathrm{A}}, \tilde{\mathrm{B}}, \tilde{\mathrm{C}}, \tilde{\mathrm{D}}, \tilde{\mathrm{E}}, \tilde{\mathrm{F}}, \tilde{\mathrm{G}}, \tilde{\mathrm{H}}, \tilde{I}\}$, then $(\tilde{\mathrm{A}}, \tilde{\tau})$ is a fuzzy gs-connected space but not fuzzy gsp-connected space.

Remark (3.21): The converse of (8) of theorem (3.6) is not true in general as shown by the following example. Example (3.22):The space $(\tilde{A}, \tilde{\tau})$ in the example 3.12 is a fuzzy gp-connected space but not fuzzy gspconnected space.

We will explain the relationship between of some types of fuzzy generalized connected in fuzzy topological space on fuzzy set by fig. (1)

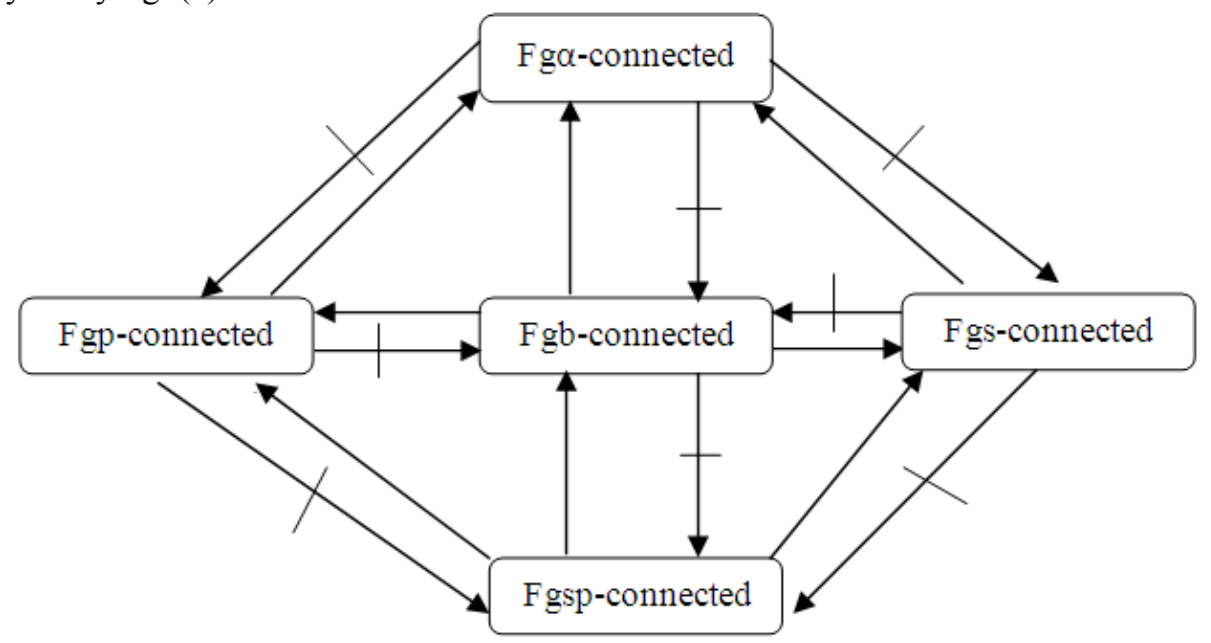

Fig.(1)

Theorem (3.23):A f.t.s( $(\tilde{\mathrm{A}}, \tilde{\mathrm{T}})$ is fuzzy gb-connected if and only if there exist nonon-empty fuzzy gb-closedsets $\tilde{E}$ EandF̃in $\tilde{A}$, such that $\mu_{\tilde{\mathrm{A}}}(\mathrm{x})=\operatorname{Max}\left\{\left(\mu_{\tilde{\mathrm{E}}}(\mathrm{x}), \mu_{\tilde{\mathrm{F}}}(\mathrm{x})\right\}\right.$ and $\operatorname{Min}\left\{\left(\mu_{\tilde{\mathrm{E}}}(\mathrm{x}), \mu_{\tilde{\mathrm{F}}}(\mathrm{x})\right\}=0\right.$.

Proof:

$\Leftrightarrow$ Suppose that $(\tilde{A}, \tilde{T})$ is fuzzy gb-connected space.

Suppose that there exists non-empty fuzzy gb-closed sets Ẽ andF̃in $\tilde{A}$, such that

$\mu_{\tilde{\mathrm{A}}}(\mathrm{x})=\operatorname{Max}\left\{\left(\mu_{\tilde{\mathrm{E}}}(\mathrm{x}), \mu_{\tilde{\mathrm{F}}}(\mathrm{x})\right\}\right.$ and $\operatorname{Min}\left\{\left(\mu_{\tilde{\mathrm{E}}}(\mathrm{x}), \mu_{\tilde{\mathrm{F}}}(\mathrm{x})\right\}=0\right.$.

Since ẼandF̃are fuzzy gb-closed sets in $\tilde{\mathrm{A}}$ and $\operatorname{Min}\left\{\left(\mu_{\tilde{\mathrm{E}}}(\mathrm{x}), \mu_{\tilde{\mathrm{F}}}(\mathrm{x})\right\}=0\right.$.

Implies that by theorem (2.6) Ẽand $\tilde{F}$ are fuzzy gb-separated sets in $\tilde{\mathrm{A}}$

Since, $\mu_{\tilde{\AA}}(\mathrm{x})=\operatorname{Max}\left\{\left(\mu_{\tilde{\mathrm{E}}}(\mathrm{x}), \mu_{\tilde{\mathrm{F}}}(\mathrm{x})\right\}\right.$

Then $(\tilde{\mathrm{A}}, \tilde{\mathrm{T}})$ is fuzzy gb-disconnected, which is a contradiction.

$(\Leftrightarrow)$ Suppose that there exists no non-empty fuzzy s-closed sets $\tilde{E}$ andF̃in $\tilde{A}$, such that $\mu_{\tilde{\mathrm{A}}}(\mathrm{x})=\operatorname{Max}\left\{\left(\mu_{\tilde{\mathrm{E}}}(\mathrm{x}), \mu_{\tilde{\mathrm{F}}}(\mathrm{x})\right\}\right.$ and $\operatorname{Min}\left\{\left(\mu_{\tilde{\mathrm{E}}}(\mathrm{x}), \mu_{\tilde{\mathrm{F}}}(\mathrm{x})\right\}=0\right.$.

Suppose that $(\tilde{\mathrm{A}}, \tilde{\mathrm{T}})$ is fuzzy $\mathrm{gb}$-disconnected space

Then this implies that there exist non-empty maximal fuzzy gb -separated sets $\tilde{\mathrm{B}}$ and $\tilde{\mathrm{C}}$ in $\tilde{\mathrm{A}}$, such that $\mu_{\tilde{\mathrm{A}}}(\mathrm{x})$ $=\operatorname{Max}\left\{\mu_{\tilde{B}}(\mathrm{x}), \mu_{\tilde{\mathrm{C}}}(\mathrm{x})\right\}$

Since $\tilde{B}$ and $\tilde{C}$ are fuzzy gb-separated sets in $\tilde{A}$

Implies that, $\operatorname{Min}\left\{\mu_{\mathrm{gbcl}(\tilde{\mathrm{B}})}(\mathrm{x}), \mu_{\tilde{\mathrm{C}}}(\mathrm{x})\right\}=0$ and $\operatorname{Min}\left\{\mu_{\mathrm{gbcl}(\tilde{\mathrm{C}})}(\mathrm{x}), \mu_{\tilde{\mathrm{B}}}(\mathrm{x})\right\}=0$

Then, $\mu_{\tilde{\mathrm{C}}}(\mathrm{x}) \leq\left[\mu_{\mathrm{gbcl}(\tilde{\mathrm{B}})}(\mathrm{x})\right]^{\mathrm{c}}$ and $\mu_{\tilde{\mathrm{B}}}(\mathrm{x}) \leq\left[\mu_{\text {gbcl }(\tilde{\mathrm{C}})}(\mathrm{x})\right]^{\mathrm{c}}$

Since $\mu_{\tilde{\mathrm{A}}}(\mathrm{x})=\operatorname{Max}\left\{\mu_{\tilde{\mathrm{B}}}(\mathrm{x}), \mu_{\tilde{\mathrm{C}}}(\mathrm{x})\right\} \leq \operatorname{Max}\left\{\left[\mu_{\mathrm{gbcl}(\tilde{\mathrm{B}})}(\mathrm{x})\right]^{\mathrm{c}},\left[\mu_{\mathrm{gbcl}(\tilde{\mathrm{C}})}(\mathrm{x})\right]^{\mathrm{c}}\right.$

Implies that, $\mu_{\tilde{\mathrm{A}}}(\mathrm{x})=\operatorname{Max}\left\{\left[\mu_{\mathrm{gbcl}(\tilde{\mathrm{B}})}(\mathrm{x})\right]^{\mathrm{c}},\left[\mu_{\mathrm{gbcl}(\tilde{\mathrm{C}})}(\mathrm{x})\right]^{\mathrm{c}}\right.$ 
Then:

$\mu_{\tilde{\mathrm{A}}}(\mathrm{x})=\operatorname{Min}\left\{\left[\mu_{\operatorname{gbcl}(\tilde{\mathrm{B}})}(\mathrm{x})\right]^{\mathrm{c}},\left[\mu_{\operatorname{gbcl}(\tilde{\mathrm{C}})}(\mathrm{x})\right]^{\mathrm{c}}\right.$

$\mu_{\tilde{\mathrm{A}}}^{\mathrm{c}}(\mathrm{x})=\operatorname{Min}\left\{\mu_{\mathrm{gbcl}(\tilde{\mathrm{B}})}(\mathrm{x}), \mu_{\mathrm{gbcl}(\tilde{\mathrm{C}})}(\mathrm{x})\right\}$

$\mu_{\tilde{\varnothing}}(\mathrm{x})=\operatorname{Min}\left\{\mu_{\mathrm{gbcl}(\tilde{\mathrm{B}})}(\mathrm{x}), \mu_{\mathrm{gbcl}(\tilde{\mathrm{C}})}(\mathrm{x})\right.$

Let $\operatorname{gb}-\operatorname{cl}(\tilde{\mathrm{C}})=\tilde{\mathrm{E}}$ and $\mathrm{gb}-\operatorname{cl}(\tilde{\mathrm{B}})=\tilde{\mathrm{F}}$

Then, $\operatorname{Min}\left\{\left(\mu_{\tilde{E}}(\mathrm{x}), \mu_{\tilde{\mathrm{F}}}(\mathrm{x})\right\}=\mu_{\tilde{\varnothing}}(\mathrm{x})\right.$

$\operatorname{Max}\left\{\left(\mu_{\tilde{\mathrm{E}}}(\mathrm{x}), \mu_{\tilde{\mathrm{F}}}(\mathrm{x})\right\}=\operatorname{Max}\left\{\mu_{\mathrm{gbcl}(\tilde{\mathrm{C}})}(\mathrm{x}), \mu_{\mathrm{gbcl}(\tilde{\mathrm{B}})}(\mathrm{x})\right\}\right.$

$\leq \mu g_{\mathrm{bcl}(\max \{} \mu_{\tilde{\mathrm{B}}(\mathrm{x}),} \mu_{\tilde{\mathrm{C}}(\mathrm{x})\})}(\mathrm{x})$

$=\mu_{\mathrm{gbcl}(\tilde{\mathrm{A}})}(\mathrm{x})=\tilde{\mathrm{A}}$

Implies that, $\operatorname{Max}\left\{\left(\mu_{\tilde{\mathrm{E}}}(\mathrm{x}), \mu_{\tilde{\mathrm{F}}}(\mathrm{x})\right\}=\mu_{\tilde{\mathrm{A}}}(\mathrm{x})\right.$, which is a contradiction.

Hence, $(\tilde{\mathrm{A}}, \tilde{\mathrm{T}})$ is fuzzy gb-connected space.

Corollary (3.24) :A f.t.s ( $\tilde{\mathrm{A}}, \tilde{\mathrm{T}})$ is fuzzy gb-connected if and only if there exist no non-empty fuzzy gb-open sets

$\tilde{\mathrm{G}}$ and $\tilde{\mathrm{H}}$ in $\tilde{\mathrm{A}}$, such that $\mu_{\tilde{\mathrm{A}}}(\mathrm{x})=\operatorname{Max}\left\{\left(\mu_{\tilde{\mathrm{G}}}(\mathrm{x}), \mu_{\tilde{\mathrm{H}}}(\mathrm{x})\right\} \operatorname{andMin}\left\{\left(\mu_{\tilde{\mathrm{G}}}(\mathrm{x}), \mu_{\tilde{\mathrm{H}}}(\mathrm{x})\right\}=\mu_{\tilde{\varnothing}}(\mathrm{x})\right.\right.$

Proof:

$\Leftrightarrow)$ Suppose that $(\tilde{A}, \tilde{T})$ is fuzzy gb-connected space.

Suppose that there exists non-empty fuzzy gb-open sets $\tilde{\mathrm{G}}$ and $\tilde{\mathrm{H}}$ in $\tilde{\mathrm{A}}$, such that $\mu_{\tilde{\mathrm{A}}}(\mathrm{x})=\operatorname{Max}\left\{\left(\mu_{\tilde{\mathrm{G}}}(\mathrm{x}), \mu_{\tilde{\mathrm{H}}}(\mathrm{x})\right\}\right.$ and $\operatorname{Min}\left\{\left(\mu_{\tilde{\mathrm{G}}}(\mathrm{x}), \mu_{\tilde{\mathrm{H}}}(\mathrm{x})\right\}=\mu_{\tilde{\Omega}}(\mathrm{x})\right.$

Then, $\mu_{\tilde{\mathrm{A}}}^{\mathrm{c}}(\mathrm{x})=\operatorname{Min}\left\{\left(\mu_{\tilde{\mathrm{G}}}^{\mathrm{c}} \mathrm{x}\right), \mu_{\tilde{\mathrm{H}}}^{\mathrm{c}}(\mathrm{x})\right\}$ and $\operatorname{Max}\left\{\left(\mu_{\tilde{\mathrm{G}}}^{\mathrm{c}}(\mathrm{x}), \mu_{\tilde{\mathrm{H}}}^{\mathrm{c}}(\mathrm{x})\right\}=\mu_{\tilde{\varnothing}}^{\mathrm{c}}(\mathrm{x})\right.$

Implies that, $\mu_{\tilde{\mathscr{\Omega}}}(\mathrm{x})=\operatorname{Min}\left\{\left(\mu_{\tilde{\mathrm{G}}}^{\mathrm{c}}(\mathrm{x}), \mu_{\tilde{\mathrm{H}}}^{\mathrm{c}} \mathrm{x}\right)\right\}$ and $\operatorname{Max}\left\{\left(\mu_{\tilde{\mathrm{G}}}^{\mathrm{c}}(\mathrm{x}), \mu_{\tilde{\mathrm{H}}}^{\mathrm{c}}(\mathrm{x})\right\}=\mu_{\tilde{\mathrm{A}}}(\mathrm{x})\right.$

Let $\tilde{\mathrm{G}}^{\mathrm{c}}=\tilde{\mathrm{E}}$ and $\tilde{\mathrm{H}}^{\mathrm{c}}=\tilde{\mathrm{F}}$, then there exist a non-empty fuzzy gb-closed sets $\tilde{\mathrm{E}}$ and $\tilde{F}$ in $\tilde{A}$, such that

$\mu_{\tilde{\mathrm{A}}}(\mathrm{x})=\operatorname{Max}\left\{\left(\mu_{\tilde{\mathrm{E}}}(\mathrm{x}), \mu_{\tilde{\mathrm{F}}}(\mathrm{x})\right\}\right.$ and $\operatorname{Min}\left\{\left(\mu_{\tilde{\mathrm{E}}}(\mathrm{x}), \mu_{\tilde{\mathrm{F}}}(\mathrm{x})\right\}=\mu_{\tilde{\Omega}}(\mathrm{x})\right.$

Then by theorem (3.23) ( $\tilde{\mathrm{A}}, \tilde{\mathrm{T}})$ is fuzzy gb-disconnected space, which is a contradiction

$(\Leftarrow)$ Suppose that there exist no non-empty fuzzy s-open sets $\tilde{\mathrm{G}}$ and $\tilde{\mathrm{H}}$ in $\tilde{\mathrm{A}}$, such that $\mu_{\tilde{\mathrm{A}}}(\mathrm{x})=\operatorname{Max}\left\{\left(\mu_{\tilde{\mathrm{G}}}(\mathrm{x}), \mu_{\tilde{\mathrm{H}}}(\mathrm{x})\right\}\right.$ and $\operatorname{Min}\left\{\left(\mu_{\tilde{\mathrm{G}}}(\mathrm{x}), \mu_{\tilde{\mathrm{H}}}(\mathrm{x})\right\}=\mu_{\tilde{\varrho}}(\mathrm{x})\right.$.

Suppose that $(\tilde{\mathrm{A}}, \tilde{T})$ is fuzzy gb-disconnected space then there exists non-empty maximal fuzzy gb-separated sets $\tilde{B}$ and $\tilde{\mathrm{C}}$ in $\tilde{\mathrm{A}}$, such that $\mu_{\tilde{\mathrm{A}}}(\mathrm{x})=\operatorname{Max}\left\{\mu_{\tilde{\mathrm{B}}}(\mathrm{x}), \mu_{\tilde{\mathrm{C}}}(\mathrm{x})\right\}$

Since $\tilde{B}$ and $\tilde{C}$ are fuzzy gb-separated sets in $\tilde{A}$

Implies that $\operatorname{Min}\left\{\mu_{\mathrm{gbcl}(\tilde{\mathrm{B}})}(\mathrm{x}), \mu_{\tilde{\mathrm{C}}}(\mathrm{x})\right\}=0$ and $\operatorname{Min}\left\{\mu_{\mathrm{gbcl}(\tilde{\mathrm{C}})}(\mathrm{x}), \mu_{\tilde{\mathrm{B}}}(\mathrm{x})\right\}=0$

Then, $\mu_{\tilde{C}}(\mathrm{x}) \leq\left[\mu_{\mathrm{gbcl}(\tilde{\mathrm{B}})}(\mathrm{x})\right]^{\mathrm{c}}$ and $\mu_{\tilde{\mathrm{B}}}(\mathrm{x}) \leq\left[\mu_{\mathrm{gbcl}(\tilde{\mathrm{C}})}(\mathrm{x})\right]^{\mathrm{c}}$

Since $\mu_{\tilde{\mathrm{A}}}(\mathrm{x})=\operatorname{Max}\left\{\mu_{\tilde{\mathrm{B}}}(\mathrm{x}), \mu_{\tilde{C}}(\mathrm{x})\right\} \leq \operatorname{Max}\left\{\left[\mu_{\mathrm{gbcl}(\tilde{\mathrm{B}})}(\mathrm{x})\right]^{\mathrm{c}},\left[\mu_{\mathrm{gbcl}(\tilde{\mathrm{C}})}(\mathrm{x})\right]^{\mathrm{c}}\right.$

Then $\mu_{\tilde{\mathrm{A}}}(\mathrm{x})=\operatorname{Max}\left\{\left[\mu_{\mathrm{gbcl}(\tilde{\mathrm{B}})}(\mathrm{x})\right]^{\mathrm{c}},\left[\mu_{\mathrm{gbcl}(\tilde{\mathrm{C}})}(\mathrm{x})\right]^{\mathrm{e}}\right.$

Let $[\operatorname{gbcl}(\tilde{\mathrm{B}})]^{\mathrm{c}}=\tilde{\mathrm{G}}$ and $[\operatorname{gbclcl}(\tilde{\mathrm{C}})]^{\mathrm{c}}=\tilde{\mathrm{H}}$

Implies that $\mu_{\tilde{\mathrm{A}}}(\mathrm{x})=\operatorname{Max}\left\{\left(\mu_{\tilde{\mathrm{G}}}(\mathrm{x}), \mu_{\tilde{\mathrm{H}}}(\mathrm{x})\right\}\right.$

$\operatorname{Min}\left\{\left(\mu_{\tilde{G}}(\mathrm{x}), \mu_{\tilde{H}}(\mathrm{x})\right\}=\operatorname{Min}\left\{\left[\mu_{\mathrm{gbcl}(\tilde{\mathrm{B}})}(\mathrm{x})\right]^{\mathrm{c}},\left[\mu_{\mathrm{gbcl}(\tilde{\mathrm{C}})}(\mathrm{x})\right]^{\mathrm{c}}\right.\right.$

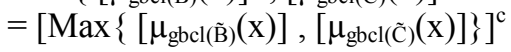

$\left.\leq\left[\mu g_{\text {bcl(max }}\left\{\mu_{\tilde{\mathrm{B}}(\mathrm{x}),} \mu_{\tilde{\mathrm{C}}}(\mathrm{x})\right\}\right)(\mathrm{x})\right]^{\mathrm{c}}$

$=\left[\mu_{\mathrm{gbcl}(\tilde{\mathrm{A}})}(\mathrm{x})\right]^{\mathrm{c}}=\mu_{\tilde{\mathrm{A}}}^{\mathrm{c}}(\mathrm{x})=\mu_{\tilde{\emptyset}}(\mathrm{x})$

Which is a contradiction.

Hence, $(\tilde{\mathrm{A}}, \tilde{\mathrm{T}})$ is fuzzy gb-connected space.

Corollary (3.25): A f.t.s ( $\tilde{\mathrm{A}}, \tilde{\mathrm{T}})$ is fuzzy weak gb-connected if and only if there exist no non-empty fuzzy gbclosed sets Ẽ andF̃in $\tilde{A}$, such that $\mu_{\tilde{\mathrm{A}}}(\mathrm{x})=\operatorname{Max}\left\{\left(\mu_{\tilde{\mathrm{E}}}(\mathrm{x}), \mu_{\tilde{\mathrm{F}}}(\mathrm{x})\right\}\right.$ and $\operatorname{Min}\left\{\left(\mu_{\tilde{\mathrm{E}}}(\mathrm{x}), \mu_{\tilde{\mathrm{F}}}(\mathrm{x})\right\}=\mu_{\tilde{\emptyset}}(\mathrm{x})\right.$

Proof :Obvious .

Corollary (3.26): A f.t.s ( $\tilde{A}, \tilde{T})$ is fuzzy weak gb-connected if and only if there exist no non-empty fuzzy s-open sets $\tilde{\mathrm{G}}$ and $\tilde{\mathrm{H}}$ in $\tilde{\mathrm{A}}$, such that $\mu_{\tilde{\mathrm{A}}}(\mathrm{x})=\operatorname{Max}\left\{\left(\mu_{\tilde{\mathrm{G}}}(\mathrm{x}), \mu_{\tilde{\mathrm{H}}}(\mathrm{x})\right\}\right.$ and $\operatorname{Min}\left\{\left(\mu_{\tilde{\mathrm{G}}}(\mathrm{x}), \mu_{\tilde{\mathrm{H}}}(\mathrm{x})\right\}=\mu_{\tilde{\varnothing}}(\mathrm{x})\right.$

Proof:Obvious .

Theorem (3.27):A f.t.s $(\tilde{\mathrm{A}}, \tilde{\mathrm{T}})$ is fuzzy gb-connected if and only if has no proper non-empty maximal fuzzy gbclopen set in $\tilde{A}$.

Proof:

$(\Rightarrow)$ Suppose that $(\tilde{\mathrm{A}}, \tilde{T})$ is fuzzy gb-connected space. Suppose that $(\tilde{\mathrm{A}}, \tilde{\mathrm{T}})$ has a proper non-empty maximal fuzzy gb-clopen set $\tilde{B}$ in $\tilde{A}$

Since $\tilde{B}$ is fuzzy gb-clopen set in $\tilde{A}$

Implies that $\tilde{\mathrm{B}}, \tilde{\mathrm{B}}^{\mathrm{c}}$ are fuzzy gb-closed sets in $\tilde{\mathrm{A}}$

Since $\tilde{B}$ is maximal fuzzy set

Then $\mu_{\tilde{\mathrm{A}}}(\mathrm{x})=\operatorname{Max}\left\{\mu_{\tilde{\mathrm{B}}}(\mathrm{x}), \mu_{\tilde{\mathrm{B}}}^{\mathrm{c}}(\mathrm{x})\right\}, \operatorname{Min}\left\{\mu_{\tilde{\mathrm{B}}}(\mathrm{x}), \mu_{\tilde{\mathrm{B}}}^{\mathrm{c}}(\mathrm{x})\right\}=\mu_{\tilde{\varnothing}}(\mathrm{x})$

$\Leftrightarrow)$ by theorem $(3.23)(\tilde{\mathrm{A}}, \tilde{\mathrm{T}})$ is fuzzy gb-disconnected space, which is a contradiction. 
$(\Leftarrow)$ Suppose that $(\tilde{\mathrm{A}}, \tilde{\mathrm{T}})$ has no proper non-empty maximal fuzzy gb-clopen set in $\tilde{\mathrm{A}}$. Suppose that $(\tilde{\mathrm{A}}, \tilde{\mathrm{T}})$ is fuzzy gb-disconnected space. Implies that, by corollary (3.24) there exist non-empty fuzzy gb-open sets $\tilde{\mathrm{G}}$ and $\tilde{\mathrm{H}}$ in $\tilde{\mathrm{A}}$, such that $\operatorname{Min}\left\{\left(\mu_{\tilde{\mathrm{G}}}(\mathrm{x}), \mu_{\tilde{\mathrm{H}}}(\mathrm{x})\right\}=\mu_{\tilde{\Phi}}(\mathrm{x})\right.$. Since $\operatorname{Min}\left\{\left(\mu_{\tilde{\mathrm{G}}}(\mathrm{x}), \mu_{\tilde{\mathrm{H}}}(\mathrm{x})\right\}=\mu_{\tilde{\varnothing}}(\mathrm{x})\right.$.

Implies that by proposition (2.5) $\tilde{\mathrm{G}} \tilde{\mathrm{q}} \tilde{\mathrm{H}}$

Since $\tilde{\mathrm{G}}$ and $\tilde{\mathrm{H}}$ are maximal fuzzy sets in $\tilde{\mathrm{A}}$

Implies that:

$\mu_{\tilde{\mathrm{G}}}(\mathrm{x})+\mu_{\tilde{\mathrm{H}}}(\mathrm{x})=\mu_{\tilde{\mathrm{A}}}(\mathrm{x})$

$\mu_{\tilde{\mathrm{G}}}(\mathrm{x})=\mu_{\tilde{\mathrm{A}}}(\mathrm{x})-\mu_{\tilde{\mathrm{H}}}(\mathrm{x})$

Hence, $\tilde{\mathrm{G}}=\tilde{\mathrm{H}}^{\mathrm{c}}$

Implies that $\tilde{\mathrm{G}}$ is fuzzy gb-clopen set in $\tilde{\mathrm{A}}$, which is a contradiction.

Corollary (3.28): A f.t.s $(\tilde{\mathrm{A}}, \tilde{\mathrm{T}})$ is fuzzy weak gb-connected if and only if there is no proper non-empty maximal fuzzy gb-clopen set in $\tilde{A}$.

Proof: Obvious.

Theorem (3.29): A f.t.s ( $\tilde{\mathrm{A}}, \tilde{\mathrm{T}})$ is fuzzy gb-connected if and only if it has no non-empty maximal fuzzy gb-open sets $\tilde{\mathrm{B}}_{1}$ and $\tilde{\mathrm{B}}_{2}$ such that $\mu_{\tilde{\mathrm{A}}}(\mathrm{x})=\operatorname{Max}\left\{\left(\mu_{\tilde{\mathrm{G}}}(\mathrm{x}), \mu_{\tilde{\mathrm{H}}}(\mathrm{x})\right\}\right.$ and $\operatorname{Min}\left\{\left(\mu_{\tilde{\mathrm{G}}}(\mathrm{x}), \mu_{\tilde{\mathrm{H}}}(\mathrm{x})\right\}=\mu_{\tilde{\varnothing}}(\mathrm{x})\right.$

$\mu_{\tilde{\mathrm{B}}_{1}}(\mathrm{x})+\mu_{\tilde{\mathrm{B}}_{2}}(\mathrm{x})=\mu_{\tilde{\mathrm{A}}}(\mathrm{x})$, for all $\mathrm{x} \in \mathrm{X}$

Proof:

$\tilde{\mathrm{A}}, \tilde{\mathrm{B}}, \tilde{\mathrm{C}}, \tilde{\mathrm{D}}, \tilde{\mathrm{E}}, \tilde{\mathrm{F}}, \tilde{\mathrm{G}}, \tilde{\mathrm{H}}$

$\Leftrightarrow$ Suppose that $(\tilde{\mathrm{A}}, \tilde{\mathrm{T}})$ is fuzzy gb-connected space. Suppose that $\tilde{\mathrm{B}}_{1}$ and $\tilde{\mathrm{B}}_{2}$ exist

Since $\mu_{\tilde{\mathrm{B}}_{1}}(\mathrm{x})+\mu_{\tilde{\mathrm{B}}_{2}}(\mathrm{x})=\mu_{\tilde{\mathrm{A}}}(\mathrm{x})$

Then, $\mu_{\tilde{B}_{1}}(\mathrm{x})=\mu_{\tilde{\mathrm{A}}}(\mathrm{x})-\mu_{\tilde{\mathrm{B}}_{2}}(\mathrm{x})=\mu_{\tilde{\mathrm{B}}_{2}^{\mathrm{c}}}(\mathrm{x})$

Hence, $\tilde{\mathrm{B}}_{1}$ is fuzzy gb-clopen set in $\tilde{\mathrm{A}}$.

Implies that by theorem $(3.27)(\tilde{\mathrm{A}}, \tilde{\mathrm{T}})$ is fuzzy gb-disconnected, which is a contradiction.

$\Leftrightarrow)$ Suppose that $(\tilde{\mathrm{A}}, \tilde{\mathrm{T}})$ has no non-empty fuzzy gb-open sets $\tilde{\mathrm{B}}_{1}$ and $\tilde{\mathrm{B}}_{2}$, such that $\mu_{\tilde{\mathrm{B}}_{1}}(\mathrm{x})+\mu_{\tilde{\mathrm{B}}_{2}}(\mathrm{x})=\mu_{\tilde{\mathrm{A}}}(\mathrm{x})$

Suppose that $(\tilde{\mathrm{A}}, \tilde{T})$ is fuzzy gb-disconnected space

Then by theorem $(3.27)(\tilde{\mathrm{A}}, \tilde{\mathrm{T}})$ has a proper fuzzy set $\tilde{\mathrm{B}}_{1}$ which is both fuzzy gb-open set and fuzzy gb-closed set. Let $\tilde{\mathrm{B}}_{2}=\tilde{\mathrm{B}}_{1}^{\mathrm{c}}$

Implies that $\tilde{\mathrm{B}}_{2}$ isfuzzy gb-openset, such that $\tilde{\mathrm{B}}_{2} \neq \tilde{\phi}$, and $\mu_{\tilde{\mathrm{B}}_{2}}(\mathrm{x})=\mu_{\tilde{\mathrm{A}}}(\mathrm{x})-\mu_{\tilde{\mathrm{B}}_{1}}(\mathrm{x})$ Then $\mu_{\tilde{\mathrm{B}}_{1}}(\mathrm{x})+\mu_{\tilde{\mathrm{B}}_{2}}(\mathrm{x})=\mu_{\tilde{\mathrm{A}}}(\mathrm{x})$, which is a contradiction.

\section{Reference}

[1]. C. L. Chang,Fuzzy Topological Spaces, J. Math. Anal. Appl.,Vol.24, 1968, 182-192.

[2]. E. Ekici, Generalization of some fuzzy functions, Institute of Mathematics Academia Sinica No.3, Vol.33, 2005.

[3]. F. S. Mahmoud,M. A. Fath Alla, and S. M. Abd Ellah, Fuzzy topology on fuzzy sets:fuzzy semicontinuity and fuzzy semisparation axioms, J. Applied Mathematics and Computation, Vol.153, 2004, 127-140.

[4]. R. Santhi and D. Jayanthi,Generalized semi-pre-connectedness in intuitionistic fuzzytopological spaces, Ann. Fuzzy Math. Inform, Vol.3, 2012, 243-253.

[5]. R. K. Sarafand N. Govindappa,On fuzzy semi-pre-generalized closed sets, J.Bull.Malays.Math. Sci.Soc.2, Vol.28,No.2, 2005, 1930.

[6]. J. A. Yusra, On Fuzzy $\alpha$-connected Space in fuzzy topological space on fuzzy set, M.Sc. Thesis, College of Education, AlMustansiryah University, 2010.

[7]. L. A. Zadeh, Fuzzy sets, Inform.Contr. 8, 1965, 338-353.

[8]. Zhen - X. Guo, and S. Fu-Gui, Connectedness based on strongly Pre-closed L-sets, Tamsui Oxford Journal of Mathematical Seiences, Vol.23,2007, 289- 304. 